\title{
The Voyager Mission Through the Jupiter Encounters
}

\author{
E. C. Stone \\ Voyager Project Scientist, California Institute of Technology, Pasadena, California 91125
}

The Voyager mission is a major element in NASA's program of exploration of the outer solar system. Objectives of the Voyager mission include comparative studies of the Jovian and Saturnian planetary systems and exploratory studies of the interplanetary medium at increasing distances from the sun. With the successful Saturn encounter in November 1980, the objectives of the mission have been extended to include an exploration of Uranus and interplanetary studies to beyond 20 astronomical units.

The papers in this special issue represent the first comprehensive report of the detailed analysis of data from the initial phase of the mission, extending from launch through the Jupiter encounters. Voyager 1 was launched on September 5, 1977, and arrived at Jupiter on March 5, 1979, while Voyager 2 was launched on August 20,1977, and arrived at Jupiter on July 9, 1979.

The two trajectories were chosen to provide complementary measurements at Jupiter within the constraints imposed by objectives at Saturn and the desire for maintaining the option for Voyager 2 to continue on to Uranus. The Voyager 1 trajectory provided reasonably close encounters with Ganymede and Callisto following closest approach to Jupiter and provided a close south polar passage at Io with an attempt to penetrate the Io flux tube, while the Voyager 2 trajectory provided a close approach to Europa and close approaches to Ganymede and Callisto prior to Jupiter closest approach. In addition, the outbound Voyager 2 trajectory allowed the deepest penetration to date of the Jovian magnetotail and both trajectories allowed earth and sun occultations for the radio and ultraviolet studies of the Jovian atmosphere. Closest approach distances to Jupiter and some of the satellites are indicated in Table 1.

Scientific studies during this initial phase included both interplanetary studies and studies of the Jovian system, which can be organized into the general areas of the atmosphere, the ring, the satellites, and the magnetosphere. The magnetosphere provides a significant coupling between these areas such that, for example, all of the scientific investigations have contributed to the study of the Io plasma torus and its effects. The scientific investigations on the two Voyager spacecraft are indicated in Table 2. A detailed description of the eleven scientific investigations, as well as of the spacecraft, the mission design, and the trajectory selection appeared in Space Science Reviews (21, 75-376, 1977).

The initial reports of the Voyager 1 encounter results were published in Science $(204,945-1006,1979)$ and in Nature (280, 725-806, 1979), followed by the initial Voyager 2 reports in Science (206, 925-995, 1979). Further analyses appeared in Geophysical Research Letters (7, 1-68, 1980). The papers in this issue reflect the status of the detailed analysis as of No-

Copyright (C) 1981 by the American Geophysical Union. vember 1980 , at which time the principal Voyager focus shifted to Saturn. These papers have, however, far from exhausted the rich veins of new information contained in the Voyager data.

The composition of the Voyager scientific teams for the first phase of the mission is as follows:

Cosmic rays. R. E. Vogt, J. R. Jokipii, F. M. McDonald, A. W. Schardt, E. C. Stone, J. H. Trainor, and W. R. Webber. Imaging science. B. A. Smith, R. F. Beebe, J. M. Boyce, G. A. Briggs, M. H. Carr, S. A. Collins, A. F. Cook II, G E. Danielson, M. E. Davies, G. E. Hunt, A. P. Ingersoll, T. V. Johnson, H. Masursky, J. F. McCauley, J. L. Mitchell, D. Morrison, T. Owen, C. Sagan, E. M. Shoemaker, L. A. Soderblom, R. G. Strom, V. E. Suomi, and J. Veverka.

Infrared radiometry and spectroscopy. R. A. Hanel, B. J. Conrath, F. M. Flasar, D. Gautier, P. Gierasch, S. Kumar, V. G. Kunde, W. C. Maguire, J. C. Pearl, J. A. Pirraglia, C. Ponnamperuma, and R. E. Samuelson.

Low-energy charged particles. S. M. Krimigis, T, P. Armstrong, W. I. Axford, C. O Bostrom, C. Y. Fan, G. Gloeckler, E. P. Keath, and L. J. Lanzerotti.

Magnetic fields. N. F. Ness, M, H. Acuna, K. W. Behannon, L. F. Burlaga, R. P. Lepping, and F. M. Neubauer.

Photopolarimetry. C. F. Lillie, C. W. Hord, D. L. Coffeen, J. E. Hansen, K. Pang, M. Sato, and R. A. West.

Planetary radio astronomy. J. W. Warwick, J. K. Alexander, A. Boischot, W. E. Brown, Jr., T. D. Carr, S. Gulkis, F. T. Haddock, C. C. Harvey, M. L. Kaiser, Y. Leblanc, J. B. Pearce, R. G. Peltzer, R. Phillips, A. C. Riddle, and D. H. Staelin

Plasma ions and electrons. H. S. Bridge, J. W. Belcher, L. F. Burlaga, C. K. Goertz, R. E. Hartle, A. J. Hundhausen, A. J. Lazarus, K. W. Ogilvie, S. Olbert, J. C. Scudder, G. L. Siscoe, J. D. Sullivan, and V. M. Vasyliunas.

Plasma waves. F. L. Scarf, D. A. Gurnett, and W S. Kurth Radio science. V. R. Eshleman, J. D. Anderson, T. A. Croft, G. S. Levy, G. F. Lindal, G. L. Tyler, and G. E. Wood. Ultraviolet spectroscopy. A. L. Broadfoot, S. K. Atreya, M. J. S. Belton, J. L. Bertaux, J. E. Blamont, A. Dalgarno, T. M. Donahue, R. Goody, J. C. McConnell, M. B. McElroy, H. W Moos, B. R. Sandel, D. E. Shemansky, and D. F Strobel.

TABLE 1. Selected Voyager Encounter Parameters

\begin{tabular}{lccr}
\hline & $\begin{array}{c}\text { Mean Distance } \\
\text { From }\end{array}$ & \multicolumn{2}{c}{ Closest Approach Distance, km } \\
\cline { 3 - 4 } \multicolumn{1}{c}{ Body } & Jupiter, $10^{3} \mathbf{~ k m ~}$ & Voyager 1 & Voyager 2 \\
\hline Jupiter & & 348,890 & 721,670 \\
Amalthea (J5) & 181.3 & 420,200 & 558,370 \\
Io (J1) & 421.6 & 20,570 & $1,129,900$ \\
Europa (J2) & 670.9 & 733,760 & 205,720 \\
Ganymede (J3) & 1070.0 & 114,710 & 62,130 \\
Callisto (J4) & 1880.0 & 126,400 & 214,930 \\
\hline
\end{tabular}

Distances are to the center of mass, not to the body surface. 


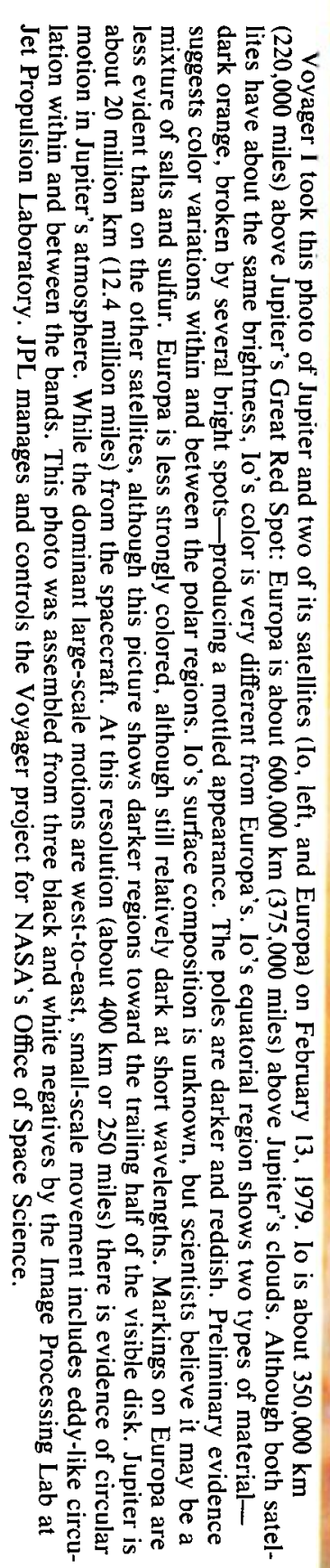


TABLE 2. Voyager Scientific Investigations

\begin{tabular}{lccl}
\hline \multicolumn{1}{c}{ Investigation } & Abbreviation & Principal Investigator/Team Leader & Institution \\
\hline Imaging science & ISS & B. A. Smith & University of Arizona \\
Infrared spectroscopy & IRIS & R. A. Hanel & Goddard Space Flight Center \\
Photopolarimetry & PPS & C. F. Lillie/C. W. Hord & University of Colorado \\
Radio science & RSS & V. R. Eshleman & Stanford University \\
Ultraviolet spectroscopy & UVS & A. L. Broadfoot & University of Southern California \\
Magnetic fields & MAG & N. F. Ness & Goddard Space Flight Center \\
Plasma ions and electrons & PLS & H. S. Bridge & Massachusetts Institute of Technology \\
Plasma waves & PWS & F. L. Scarf & TRW Defense and Space Systems \\
Planetary radio astronomy & PRA & J. W. Warwick & Radiophysics, Inc. \\
Low-energy charged particles & LECP & S. M. Krimigis & Johns Hopkins University/Applied \\
& & & Physics Laboratory \\
Cosmic rays & CRS & R. E. Vogt & California Institute of Technology \\
\hline
\end{tabular}

(Received March 13, 1981; 